\title{
Median-Type John-Nirenberg Space in Metric Measure Spaces
}

\section{Kim Myyryläinen ${ }^{1}$}

Received: 1 July 2021 / Accepted: 12 January 2022 / Published online: 4 February 2022

(c) The Author(s) 2022

\begin{abstract}
We study the so-called John-Nirenberg space that is a generalization of functions of bounded mean oscillation in the setting of metric measure spaces with a doubling measure. Our main results are local and global John-Nirenberg inequalities, which give weak-type estimates for the oscillation of a function. We consider medians instead of integral averages throughout, and thus functions are not a priori assumed to be locally integrable. Our arguments are based on a Calderón-Zygmund decomposition and a good- $\lambda$ inequality for medians. A John-Nirenberg inequality up to the boundary is proven by using chaining arguments. As a consequence, the integral-type and the median-type John-Nirenberg spaces coincide under a Boman-type chaining assumption.
\end{abstract}

Keywords John-Nirenberg space $\cdot$ Median $\cdot$ John-Nirenberg inequality $\cdot$ Doubling measure $\cdot$ Metric space

Mathematics Subject Classification 42B35 · 43A85

\section{Introduction}

The space of functions of bounded mean oscillation (BMO) was introduced by John and Nirenberg in [27]. In that article, they also discussed a larger BMO-type space called the John-Nirenberg space, denoted by $J N_{p}$ with $1<p<\infty$. The JohnNirenberg space contains BMO, and BMO is obtained as the limit of $J N_{p}$ as $p \rightarrow \infty$. The John-Nirenberg lemma in [27] states that a logarithmic blowup is the worst possible for a BMO function. Moreover, another version of the John-Nirenberg lemma in [27] implies that $J N_{p}$ is a subset of weak $L^{p}$. This inclusion is strict, as shown by

Kim Myyryläinen

kim.myyrylainen@aalto.fi

1 Department of Mathematics, Aalto University, P.O. Box 11100, 00076 Aalto, Finland 
a one-dimensional example in [1]. It is also known that $L^{p}$ is a proper subset of $J N_{p}$; see [8].

In [26], John discussed BMO using medians instead of integral averages. He also proved the analogous John-Nirenberg inequality for the median-type BMO in a Euclidean space. Later, Strömberg proved the inequality for a larger class of medians and also gave a proof in a metric measure space with a doubling measure [35, 36]. In particular, this implies that the ordinary $\mathrm{BMO}$ and the median-type $\mathrm{BMO}$ are equivalent. While BMO has been studied extensively, the John-Nirenberg space is not equally well understood. However, some results related to John-Nirenberg inequalities and interpolation of operators can be found in $[6,12-14,34]$.

Our main results are John-Nirenberg inequalities for the median-type JohnNirenberg space. We carry out the analysis in a metric measure space with a doubling measure, but the main results are new even in the Euclidean setting. The main novelty is that we consider medians instead of integral averages throughout. One of the advantages of using medians is that we do not need to assume that the function is locally integrable. Thus, the definition via medians applies to any measurable function. There exist different definitions of John-Nirenberg spaces in metric measure spaces; see [1, $2,32]$. The difference is whether the balls in the definition are required to be pairwise disjoint or allowed to overlap. Definitions with disjoint balls can be found in [2,32] and with overlap in [1]. We adopt the definition in [2,32] which leads to a more general theory.

The proof of the local John-Nirenberg inequality with medians (Theorem 4.4) in Sect. 4 is based on a Calderón-Zygmund decomposition (Lemma 4.2) and a good- $\lambda$ inequality (Lemma 4.3) for medians. This is inspired by [1,2], where the authors examine integral-type John-Nirenberg spaces in metric measure spaces with a doubling measure. A challenge in proving results for the median-type John-Nirenberg space is that medians lack subadditive properties and monotonicity on sets compared to integral averages. Our Theorem 4.4 implies a related John-Nirenberg inequality in [30,Theorem 1.3] where a discrete summability condition is considered; see also [2, $10,31]$. A simple one-dimensional example shows that our result is more general than [30,Theorem 1.3]. In particular, our approach does not depend on the discrete summability condition and rearrangements.

In Sect. 5, we prove a John-Nirenberg inequality with medians up to the boundary (Theorem 5.3) in Boman sets by applying the local John-Nirenberg inequality together with chaining arguments. Boman sets are closely related to the Boman chain condition, introduced in the unpublished paper [4]. The connection is discussed in [32]. In particular, the two are equivalent in a geodesic space with a doubling measure. The Boman chain condition characterizes John domains in many metric measure spaces, including Euclidean spaces; see [5]. The corresponding results for the integral-type John-Nirenberg space can be found in [23, 32]. We apply the arguments in [7, 23, 32] for medians. As a corollary of the global John-Nirenberg inequality, we show that the integral- and median-type John-Nirenberg spaces coincide in every open set under the assumption that balls are Boman sets with uniform parameters (Corollary 5.4). This means that the median-type John-Nirenberg condition is possibly the weakest for a function to be in $J N_{p}$. The uniform Boman condition on balls holds, for example, in geodesic spaces [16]. 


\section{Preliminaries}

Let $(X, d, \mu)$ be a metric measure space with a metric $d$ and a doubling measure $\mu$. A Borel regular measure is said to be doubling if

$$
0<\mu(2 B) \leq c_{\mu} \mu(B)<\infty
$$

for every ball $B=B(x, r)=\{y \in X: d(x, y)<r\}$, where $c_{\mu}>1$ is the doubling constant. We use the notation $\lambda B=B(x, \lambda r), \lambda>0$, for the $\lambda$-dilate of $B$. From the doubling property of the measure, it can be deduced that if $y \in B(x, R) \subset X$ and $0<r \leq R<\infty$, then

$$
\frac{\mu(B(x, R))}{\mu(B(y, r))} \leq c_{\mu}^{2}\left(\frac{R}{r}\right)^{D},
$$

where $D=\log _{2} c_{\mu}$ is the doubling dimension of the space $(X, d, \mu)$. The proof can be found in [3,p. 6]. We denote the integral average of a function $f \in L^{1}(A)$ in a set $A \subset X$ by

$$
f_{A}=f_{A} f d \mu=\frac{1}{\mu(A)} \int_{A} f d \mu \text {. }
$$

A basic tool in metric measure spaces is the 5-covering theorem. It is also sometimes referred to as the basic covering theorem or Vitali's covering theorem. Although, one must be careful since there is another covering theorem named after Vitali [22,pp. 3-4]. One can check [22,pp. 2-3] for a proof.

Lemma 2.1 Let $\mathcal{F}$ be a collection of balls of uniformly bounded radii in $X$. Then there exists a countable disjointed subcollection $\mathcal{G}$ such that

$$
\bigcup_{B \in \mathcal{F}} B \subset \bigcup_{B \in \mathcal{G}} 5 B
$$

The Lebesgue differentiation theorem states that the integral average of a function $f$ over a ball $B(x, r)$ approaches $f(x)$ when the radius $r$ tends to zero. Two different proofs can be found in [22,pp. 4-6, 12-13].

Lemma 2.2 If $f \in L_{\text {loc }}^{1}(X)$, then it holds that

$$
\lim _{r \rightarrow 0} f_{B(x, r)}|f-f(x)| d \mu=0
$$

for $\mu$-almost every $x \in X$.

We follow the definition in $[2,32]$ for the integral-type John-Nirenberg space $J N_{p, q}$ in metric measure spaces. 
Definition 2.3 Let $\Omega \subset X$ be an open set, $1<p<\infty$ and $0<q<p$. We say that a function $f \in L_{\mathrm{loc}}^{q}(\Omega)$ belongs to the John-Nirenberg space $J N_{p, q}(\Omega)$ if

$$
\|f\|_{J N_{p, q}(\Omega)}^{p}=\sup \sum_{i=1}^{\infty} \mu\left(B_{i}\right)\left(\inf _{c_{i} \in \mathbb{R}} f_{B_{i}}\left|f-c_{i}\right|^{q} d \mu\right)^{\frac{p}{q}}<\infty
$$

where the supremum is taken over countable collections of pairwise disjoint balls $B_{i} \subset \Omega$.

If $q=1$, we write $J N_{p}$ instead of $J N_{p, 1}$. We omit $\Omega$ from the norms if the considered set is clear from the context.

Next, we present medians and discuss their properties. Medians have been studied and used in different problems of analysis; see for example [9, 11, 15, 17-21, 24-26, 28-30, 33, 35-37].

Definition 2.4 Let $A \subset X$ be a set of finite and positive measure, $0<s \leq 1$ and $f: X \rightarrow[-\infty, \infty]$ be a measurable function. If a value $M_{f}^{s}(A)$ satisfies

$$
\mu\left(\left\{x \in A: f(x)>M_{f}^{s}(A)\right\}\right) \leq s \mu(A)
$$

and

$$
\mu\left(\left\{x \in A: f(x)<M_{f}^{s}(A)\right\}\right) \leq(1-s) \mu(A),
$$

then we call $M_{f}^{s}(A)$ an $s$-median of the function $f$ over a set $A$.

Note that a $\frac{1}{2}$-median is a standard median value of $f$ over $A$. An $s$-median of a function is not always unique. For example, consider $f=\chi_{[1 / 2,1]}$ on the interval $[0,1]$. Then any value between 0 and 1 is a $\frac{1}{2}$-median of $f$. Thus, we define the maximal $s$-median $m_{f}^{s}(A)$ which is unique [33].

Definition 2.5 Let $0<s \leq 1$ and $A \subset X$ be such that $0<\mu(A)<\infty$. The maximal $s$-median of a measurable function $f: X \rightarrow[-\infty, \infty]$ over a set $A$ is defined as

$$
m_{f}^{s}(A)=\inf \{a \in \mathbb{R}: \mu(\{x \in A: f(x)>a\})<s \mu(A)\} .
$$

It can be shown that the maximal $s$-median of a function is indeed an $s$-median [33]. In the next lemma, we list the basic properties of the maximal $s$-median. Most of these properties are listed without proofs in [20, 21]. The proofs of properties (i), (ii), (v), (vii), (viii) and (ix) can be found in [33,Proposition 1.1] in the Euclidean setting. The proofs of these properties are practically same in metric measure spaces, and thus are omitted here. We give proofs for the remaining properties.

Lemma 2.6 Let $A \subset X$ such that $0<\mu(A)<\infty, f, g: X \rightarrow[-\infty, \infty]$ be measurable functions and $0<s \leq 1$. Then the maximal $s$-median has the following properties: 
(i) If $s \leq s^{\prime}$, then $m_{f}^{s^{\prime}}(A) \leq m_{f}^{s}(A)$.

(ii) $m_{f}^{s}(A) \leq m_{g}^{s}(A)$ whenever $f \leq g \mu$-almost everywhere in $A$.

(iii) If $A \subset A^{\prime}$ and $\mu\left(A^{\prime}\right) \leq c \mu(A)$ with some $c \geq 1$, then $m_{f}^{s}(A) \leq m_{f}^{s / c}\left(A^{\prime}\right)$.

(iv) $m_{\varphi \circ f}^{s}(A)=\varphi\left(m_{f}^{s}(A)\right)$ for an increasing continuous function $\varphi: f[A] \rightarrow$ $[-\infty, \infty]$.

(v) $m_{f}^{s}(A)+c=m_{f+c}^{s}$ (A) for $c \in \mathbb{R}$.

(vi) $m_{c f}^{s}(A)=c m_{f}^{s}(A)$ for $c>0$.

(vii) $\left|m_{f}^{s}(A)\right| \leq m_{|f|}^{\min \{s, 1-s\}}(A)$.

(viii) $m_{f+g}^{s}(A) \leq m_{f}^{t_{1}}(A)+m_{g}^{t_{2}}(A)$ whenever $t_{1}+t_{2} \leq s$.

(ix) For $f \in L^{p}(A)$ and $p>0$,

$$
m_{|f|}^{s}(A) \leq\left(s^{-1} f_{A}|f|^{p} d \mu\right)^{\frac{1}{p}}
$$

(x) If $A_{i}$ are pairwise disjoint for every $i \in \mathbb{N}$, then

$$
\inf _{i} m_{f}^{s}\left(A_{i}\right) \leq m_{f}^{s}\left(\bigcup_{i=1}^{\infty} A_{i}\right) \leq \sup _{i} m_{f}^{s}\left(A_{i}\right) .
$$

Proof (iii) If $a>m_{f}^{s / c}\left(A^{\prime}\right)$, then we have

$$
\mu(\{x \in A: f(x)>a\}) \leq \mu\left(\left\{x \in A^{\prime}: f(x)>a\right\}\right)<\frac{s}{c} \mu\left(A^{\prime}\right) \leq s \mu(A),
$$

from which the claim follows.

(iv) It holds that

$$
\begin{aligned}
\varphi\left(m_{f}^{s}(A)\right) & =\inf \{\varphi(a) \in \mathbb{R}: \mu(\{x \in A: f(x)>a\})<s \mu(A)\} \\
& =\inf \{\varphi(a) \in \mathbb{R}: \mu(\{x \in A: \varphi(f(x))>\varphi(a)\})<s \mu(A)\} \\
& =m_{\varphi \circ f}^{s}(A) .
\end{aligned}
$$

(vi) Apply property (iv) for $\varphi(x)=c x$.

(x) For $a>\sup _{i} m_{f}^{s}\left(A_{i}\right)$, we have

$$
\begin{aligned}
\mu\left(\left\{x \in \bigcup_{i=1}^{\infty} A_{i}: f(x)>a\right\}\right) & =\sum_{i=1}^{\infty} \mu\left(\left\{x \in A_{i}: f(x)>a\right\}\right) \\
& <\sum_{i=1}^{\infty} s \mu\left(A_{i}\right)=s \mu\left(\bigcup_{i=1}^{\infty} A_{i}\right)
\end{aligned}
$$


It follows that $m_{f}^{s}\left(\bigcup_{i=1}^{\infty} A_{i}\right) \leq a$. Since this holds for every $a>\sup _{i} m_{f}^{s}\left(A_{i}\right)$, we have

$$
m_{f}^{s}\left(\bigcup_{i=1}^{\infty} A_{i}\right) \leq \sup _{i} m_{f}^{s}\left(A_{i}\right) .
$$

To prove the other inequality, assume that $a<\inf _{i} m_{f}^{s}\left(A_{i}\right)$. We then get

$$
\begin{aligned}
\mu\left(\left\{x \in \bigcup_{i=1}^{\infty} A_{i}: f(x)<a\right\}\right) & =\sum_{i=1}^{\infty} \mu\left(\left\{x \in A_{i}: f(x)<a\right\}\right) \\
& <\sum_{i=1}^{\infty}(1-s) \mu\left(A_{i}\right)=(1-s) \mu\left(\bigcup_{i=1}^{\infty} A_{i}\right) .
\end{aligned}
$$

This implies that $a \leq m_{f}^{s}\left(\bigcup_{i=1}^{\infty} A_{i}\right)$ for every $a<\inf _{i} m_{f}^{s}\left(A_{i}\right)$. Hence, we obtain

$$
\inf _{i} m_{f}^{s}\left(A_{i}\right) \leq m_{f}^{s}\left(\bigcup_{i=1}^{\infty} A_{i}\right) .
$$

Remark Assume that $0<s \leq 1 / 2$. Then property (vii) assumes a slightly simpler form

$$
\left|m_{f}^{s}(A)\right| \leq m_{|f|}^{\min \{s, 1-s\}}(A)=m_{|f|}^{s}(A)
$$

since

$$
m_{|f|}^{1-s}(A) \leq m_{|f|}^{s}(A)
$$

for $0<s \leq 1 / 2$.

Suppose that $f \in L_{\mathrm{loc}}^{1}(X)$ and $0<s \leq 1 / 2$. Using the Lebesgue differentiation theorem (Lemma 2.2) together with properties (v), (vii) and (ix) of the maximal $s$ medians, we obtain the following version of the Lebesgue differentiation theorem:

$$
\begin{aligned}
\left|m_{f}^{s}(B(x, r))-f(x)\right| & =\left|m_{f-f(x)}^{s}(B(x, r))\right| \\
& \leq m_{|f-f(x)|}^{s}(B(x, r)) \\
& \leq s^{-1} f_{B(x, r)}|f-f(x)| d \mu \rightarrow 0
\end{aligned}
$$

as $r \rightarrow 0$. However, there is a more general version of the Lebesgue differentiation theorem for medians [33], where we need to assume only that $f$ is a measurable function. 
The proof of Lemma 2.7 can be found in [33,Theorem 2.1] where the lemma is proven in the Euclidean setting. The proof is almost identical in metric measure spaces with a doubling measure, and thus is omitted here.

Lemma 2.7 Let $f: X \rightarrow[-\infty, \infty]$ be a measurable function which is finite almost everywhere in $X$ and $0<s \leq 1$. Then it holds that

$$
\lim _{r \rightarrow 0} m_{f}^{s}(B(x, r))=f(x)
$$

for $\mu$-almost every $x \in X$.

We give a definition for the median-type $\mathrm{BMO}$ which coincides with the classical BMO [26, 35, 36].

Definition 2.8 We say that a measurable function $f$ belongs to $\mathrm{BMO}_{0, s}(X)$ if

$$
\|f\|_{\mathrm{BMO}_{0, s}(X)}=\sup _{B \subset X} \inf _{c} m_{|f-c|}^{s}(B)<\infty .
$$

\section{Definition and Properties of $J N_{p, 0, s}$}

In this section, we give a definition of the median-type John-Nirenberg space in metric measure spaces. Moreover, we examine the basic properties of the space.

Definition 3.1 Let $\Omega \subset X$ be an open set, $1<p<\infty$ and $0<s \leq 1 / 2$. We say that a measurable function $f$ belongs to the median-type John-Nirenberg space $J N_{p, 0, s}(\Omega)$ if

$$
\|f\|_{J N_{p, 0, s}(\Omega)}^{p}=\sup \sum_{i=1}^{\infty} \mu\left(B_{i}\right)\left(\inf _{c_{i} \in \mathbb{R}} m_{\left|f-c_{i}\right|}^{s}\left(B_{i}\right)\right)^{p}<\infty,
$$

where the supremum is taken over all countable collections of pairwise disjoint balls $B_{i} \subset \Omega$.

The zero in the notation $J N_{p, 0, s}$ means that we do not need to assume any local integrability but only measurability. The range $0<s \leq 1 / 2$ is necessary since $\|f\|_{J N_{p, 0, s}}=0$ for $s>1 / 2$ and a two-valued function $f$.

The next lemma shows that the constants $c_{i}$ in the definition of $J N_{p, 0, s}$ can be replaced by the maximal $t$-medians where $s \leq t \leq \frac{1}{2}$.

Lemma 3.2 Let $f$ be a measurable function. It holds that

$$
\|f\|_{J N_{p, 0, s}(\Omega)}^{p} \leq \sup \sum_{i=1}^{\infty} \mu\left(B_{i}\right)\left(m_{\left|f-m_{f}^{t}\left(B_{i}\right)\right|}^{s}\left(B_{i}\right)\right)^{p} \leq 2^{p}\|f\|_{J N_{p, 0, s}(\Omega)}^{p},
$$

whenever $0<s \leq t \leq 1 / 2$. 
Proof It is clear that the first inequality holds. The other inequality follows from

$$
\begin{aligned}
m_{\left|f-m_{f}^{t}\left(B_{i}\right)\right|}^{s}\left(B_{i}\right) & \leq m_{\left|f-c_{i}\right|+\left|m_{f}^{t}\left(B_{i}\right)-c_{i}\right|}^{s}\left(B_{i}\right) \\
& =m_{\left|f-c_{i}\right|}^{s}\left(B_{i}\right)+\left|m_{f}^{t}\left(B_{i}\right)-c_{i}\right| \\
& =m_{\left|f-c_{i}\right|}^{s}\left(B_{i}\right)+\left|m_{f-c_{i}}^{t}\left(B_{i}\right)\right| \\
& \leq m_{\left|f-c_{i}\right|}^{s}\left(B_{i}\right)+m_{\left|f-c_{i}\right|}^{t}\left(B_{i}\right) \\
& \leq 2 m_{\left|f-c_{i}\right|}^{s}\left(B_{i}\right) .
\end{aligned}
$$

By taking the infimum over the constants $c_{i}$, we observe that

$$
m_{\left|f-m_{f}^{t}\left(B_{i}\right)\right|}^{s}\left(B_{i}\right) \leq 2 \inf _{c_{i}} m_{\left|f-c_{i}\right|}^{s}\left(B_{i}\right) .
$$

Therefore, the second inequality is attained as well.

Next, we list some basic properties of $J N_{p, 0, s}$ spaces.

Lemma 3.3 Let $f$ and $g$ be measurable functions. Then the following properties hold true:

(i) $\|f+g\|_{J N_{p, 0, s}} \leq\|f\|_{J N_{p, 0, t_{1}}}+\|g\|_{J N_{p, 0, t_{2}}}$ whenever $t_{1}+t_{2} \leq s$.

(ii) $\||f|\|_{J N_{p, 0, s}} \leq\|f\|_{J N_{p, 0, s}}$.

(iii) If $2 t_{1}+2 t_{2} \leq s$, then

$$
\begin{aligned}
\|\max \{f, g\}\|_{J N_{p, 0, s}} & \leq\|f\|_{J N_{p, 0, t_{1}}}+\|g\|_{J N_{p, 0, t_{2}}}, \\
\|\min \{f, g\}\|_{J N_{p, 0, s}} & \leq\|f\|_{J N_{p, 0, t_{1}}}+\|g\|_{J N_{p, 0, t_{2}}} .
\end{aligned}
$$

Proof (i) Using property (viii) of Lemma 2.6 and Minkowski's inequality, we estimate

$$
\begin{aligned}
& {\left[\sum_{i=1}^{\infty} \mu\left(B_{i}\right)\left(\inf _{c_{i}} m_{\left|f+g-c_{i}\right|}^{s}\left(B_{i}\right)\right)^{p}\right]^{\frac{1}{p}}} \\
& \leq\left[\sum_{i=1}^{\infty} \mu\left(B_{i}\right)\left(m_{\left|f+g-c_{i}^{f}-c_{i}^{g}\right|}^{s}\left(B_{i}\right)\right)^{p}\right]^{\frac{1}{p}} \\
& \leq\left[\sum_{i=1}^{\infty} \mu\left(B_{i}\right)\left(m_{\left|f-c_{i}^{f}\right|}^{t_{1}}\left(B_{i}\right)+m_{\left|g-c_{i}^{g}\right|}^{t_{2}}\left(B_{i}\right)\right)^{p}\right]^{\frac{1}{p}} \\
& =\left[\int_{\Omega}\left(\sum_{i=1}^{\infty} \chi_{B_{i}}(x) m_{\left|f-c_{i}^{f}\right|}^{t_{1}}\left(B_{i}\right)+\sum_{i=1}^{\infty} \chi_{B_{i}}(x) m_{\left|g-c_{i}^{g}\right|}^{t_{2}}\left(B_{i}\right)\right)^{p} d \mu(x)\right]^{\frac{1}{p}} \\
& \leq\left[\int_{\Omega}\left(\sum_{i=1}^{\infty} \chi_{B_{i}}(x) m_{\left|f-c_{i}^{f}\right|}^{t_{1}}\left(B_{i}\right)\right)^{p} d \mu(x)\right]^{\frac{1}{p}}+\left[\int_{\Omega}\left(\sum_{i=1}^{\infty} \chi_{B_{i}}(x) m_{\left|g-c_{i}^{g}\right|}^{t_{2}}\left(B_{i}\right)\right)^{p} d \mu(x)\right]^{\frac{1}{p}} \\
& =\left[\sum_{i=1}^{\infty} \mu\left(B_{i}\right)\left(m_{\left|f-c_{i}^{f}\right|}^{t_{1}}\left(B_{i}\right)\right)^{p}\right]^{\frac{1}{p}}+\left[\sum_{i=1}^{\infty} \mu\left(B_{i}\right)\left(m_{\left|g-c_{i}^{g}\right|}^{t_{2}}\left(B_{i}\right)\right)^{p}\right]^{\frac{1}{p}}
\end{aligned}
$$


for any $c_{i}^{f}, c_{i}^{g} \in \mathbb{R}$. Thus, we can take the infimum over the constants $c_{i}^{f}$ and $c_{i}^{g}$ to get

$$
\|f+g\|_{J N_{p, 0, s}} \leq\|f\|_{J N_{p, 0, t_{1}}}+\|g\|_{J N_{p, 0, t_{2}}},
$$

where $t_{1}+t_{2} \leq s$.

(ii) The triangle inequality together with Lemma 2.6 (ii) implies

$$
\begin{aligned}
\sum_{i=1}^{\infty} \mu\left(B_{i}\right)\left(\inf _{c_{i}} m_{|| f\left|-c_{i}\right|}^{s}\left(B_{i}\right)\right)^{p} & \leq \sum_{i=1}^{\infty} \mu\left(B_{i}\right)\left(\inf _{c_{i}} m_{|| f|-| c_{i}||}^{s}\left(B_{i}\right)\right)^{p} \\
& \leq \sum_{i=1}^{\infty} \mu\left(B_{i}\right)\left(\inf _{c_{i}} m_{\left|f-c_{i}\right|}^{s}\left(B_{i}\right)\right)^{p}
\end{aligned}
$$

Therefore, we obtain the claim

$$
\||f|\|_{J N_{p, 0, s}} \leq\|f\|_{J N_{p, 0, s}} .
$$

(iii) Note that $\max \{f, g\}=\frac{1}{2}(f+g+|f-g|)$ and $\min \{f, g\}=\frac{1}{2}(f+g-|f-g|)$. Then using (i) and (ii), we get

$$
\begin{aligned}
\|\max \{f, g\}\|_{J N_{p, 0, s}} & \leq \frac{1}{2}\left(\|f+g\|_{J N_{p, 0, t_{1}+t_{2}}}+\|\mid f-g\|_{J N_{p, 0, t_{1}+t_{2}}}\right) \\
& \leq \frac{1}{2}\left(\|f\|_{J N_{p, 0, t_{1}}}+\|g\|_{J N_{p, 0, t_{2}}}+\|f-g\|_{J N_{p, 0, t_{1}+t_{2}}}\right) \\
& \leq \frac{1}{2}\left(\|f\|_{J N_{p, 0, t_{1}}}+\|g\|_{J N_{p, 0, t_{2}}}+\|f\|_{J N_{p, 0, t_{1}}}+\|g\|_{J N_{p, 0, t_{2}}}\right) \\
& =\|f\|_{J N_{p, 0, t_{1}}}+\|g\|_{J N_{p, 0, t_{2}}},
\end{aligned}
$$

where $2 t_{1}+2 t_{2} \leq s$. The claim for $\min \{f, g\}$ follows similarly.

The next proposition tells that the space $L^{p}$ is contained in $J N_{p, q}$ which in turn is a subset of $J N_{p, 0, s}$. The first inclusion is strict in the Euclidean setting, that is, there exists a function in $J N_{p} \backslash L^{p}$ [8]. The second one holds in the other direction in many situations; see Corollary 5.4.

Proposition 3.4 Let $1<p<\infty, 0<q<p$ and $0<s \leq 1 / 2$. It holds that $L^{p}(\Omega) \subset J N_{p, q}(\Omega) \subset J N_{p, 0, s}(\Omega)$, particularly

$$
s^{\frac{1}{q}}\|f\|_{J N_{p, 0, s}(\Omega)} \leq\|f\|_{J N_{p, q}(\Omega)} \leq\|f\|_{L^{p}(\Omega)} .
$$


Proof The first inequality follows straightforwardly from property (ix) of Lemma 2.6. The second one is obtained by a simple use of Hölder's inequality:

$$
\begin{aligned}
\sum_{i=1}^{\infty} \mu\left(B_{i}\right)\left(\inf _{c_{i}} f_{B_{i}}\left|f-c_{i}\right| d \mu\right)^{p} & \leq \sum_{i=1}^{\infty} \mu\left(B_{i}\right)\left(f_{B_{i}}|f| d \mu\right)^{p} \\
& \leq \sum_{i=1}^{\infty} \mu\left(B_{i}\right) f_{B_{i}}|f|^{p} d \mu \\
& \leq \int_{\Omega}|f|^{p} d \mu .
\end{aligned}
$$

By taking the supremum over all collections of pairwise disjoint balls $B_{i} \subset \Omega$, we get

$$
\|f\|_{J N_{p, q}(\Omega)} \leq\|f\|_{L^{p}(\Omega)}
$$

Remark If $f \in \mathrm{BMO}_{0, s}(\Omega)$ with $\mu(\Omega)<\infty$, then it holds that $f \in J N_{p, 0, s}(\Omega)$. More precisely, we have

$$
\|f\|_{J N_{p, 0, s}(\Omega)} \leq \mu(\Omega)^{\frac{1}{p}}\|f\|_{\mathrm{BMO}_{0, s}(\Omega)} .
$$

The previous inequality follows from the estimates

$$
\sum_{i=1}^{\infty} \mu\left(B_{i}\right)\left(\inf _{c_{i}} m_{\left|f-c_{i}\right|}^{s}\left(B_{i}\right)\right)^{p} \leq \sum_{i=1}^{\infty} \mu\left(B_{i}\right)\|f\|_{\mathrm{BMO}_{0, s}}^{p} \leq \mu(\Omega)\|f\|_{\mathrm{BMO}_{0, s}}^{p} .
$$

The median-type John-Nirenberg space $J N_{p, 0, s}$ is a generalization of BMO in the sense that a function is in BMO if and only if the $J N_{p, 0, s}$ norm of the function is uniformly bounded as $p$ tends to infinity.

Proposition 3.5 If $\Omega \subset X$ has finite measure, then it holds that

$$
\lim _{p \rightarrow \infty}\|f\|_{J N_{p, 0, s}(\Omega)}=\|f\|_{B M O_{0, s}(\Omega)}
$$

Proof Let $\left\{B_{i}\right\}_{i}$ be a collection of pairwise disjoint balls contained in $\Omega$. Remind that if $\mu(A)<\infty$, then $\|g\|_{L^{p}(A)} \rightarrow\|g\|_{L^{\infty}(A)}$ as $p \rightarrow \infty$. By using this, it follows that

$$
\begin{aligned}
\left(\sum_{i=1}^{\infty} \mu\left(B_{i}\right)\left(\inf _{c_{i}} m_{\left|f-c_{i}\right|}^{s}\left(B_{i}\right)\right)^{p}\right)^{\frac{1}{p}} & =\left(\int_{\Omega}\left(\sum_{i=1}^{\infty} \chi_{B_{i}}(x) \inf _{c_{i}} m_{\left|f-c_{i}\right|}^{s}\left(B_{i}\right)\right)^{p} d \mu(x)\right)^{\frac{1}{p}} \\
& \rightarrow \sup _{x \in \Omega} \sum_{i=1}^{\infty} \chi_{B_{i}}(x) \inf _{c_{i}} m_{\left|f-c_{i}\right|}^{s}\left(B_{i}\right) \\
& =\sup _{i} \inf _{c_{i}} m_{\left|f-c_{i}\right|}^{s}\left(B_{i}\right)
\end{aligned}
$$


as $p \rightarrow \infty$. Hence, we have

$$
\begin{aligned}
\sup _{\left\{B_{i}\right\}} \lim _{p \rightarrow \infty}\left(\sum_{i=1}^{\infty} \mu\left(B_{i}\right)\left(\inf _{c_{i}} m_{\left|f-c_{i}\right|}^{s}\left(B_{i}\right)\right)^{p}\right)^{\frac{1}{p}} & =\sup _{\left\{B_{i}\right\}} \sup _{i} \inf _{c_{i}} m_{\left|f-c_{i}\right|}^{s}\left(B_{i}\right) \\
& =\sup _{B \subset \Omega} \inf _{c} m_{|f-c|}^{s}(B) \\
& =\|f\|_{\mathrm{BMO}_{0, s} .}
\end{aligned}
$$

We can interchange the order of taking the supremum and the limit since

$$
\left(\int_{\Omega}\left(\sum_{j=1}^{\infty} \chi_{B_{j}}(x) \inf _{c_{j}} m_{\left|f-c_{j}\right|}^{s}\left(B_{j}\right)\right)^{p} d \mu(x)\right)^{\frac{1}{p}}
$$

is an increasing function of $p$ which can be seen by Hölder's inequality. Thus, we conclude that

$$
\lim _{p \rightarrow \infty}\|f\|_{J N_{p, 0, s}}=\|f\|_{\mathrm{BMO}_{0, s}} .
$$

\section{John-Nirenberg Lemma for $J N_{p, 0, s}$}

We need two lemmas to prove the John-Nirenberg inequality for $J N_{p, 0, s}$ which implies that $J N_{p, 0, s}$ is contained in $L^{p, \infty}$, that is, weak $L^{p}$. The first lemma is a Calderón-Zygmund decomposition and the second one is a good- $\lambda$ inequality.

Throughout the argument let $\eta>0$ and $B_{0}=B\left(x_{B_{0}}, r_{B_{0}}\right) \subset X$ be fixed. We denote $\widehat{B}_{0}=(1+\eta) B_{0}$,

$$
\mathcal{B}=\left\{B\left(x_{B}, r_{B}\right): x_{B} \in B_{0}, r_{B} \leq \eta r_{B_{0}}\right\}
$$

and

$$
\alpha=5^{D} c_{\mu}^{2}\left(1+\frac{1}{\eta}\right)^{D},
$$

where $c_{\mu}$ is the doubling constant and $D=\log _{2} c_{\mu}$ is the doubling dimension. We define a maximal function

$$
M_{\mathcal{B}} f(x)=\sup _{\substack{B \ni x \\ B \in \mathcal{B}}} m_{|f|}^{t}(B)
$$

with the understanding that $M_{\mathcal{B}} f(x)=0$ if there is no ball $B \in \mathcal{B}$ such that $x \in B$. In particular, $M_{\mathcal{B}} f(x)=0$ for every $x \in X \backslash \widehat{B}_{0}$. By the Lebesgue differentiation 
theorem for medians (Lemma 2.7), we have $|f(x)| \leq M_{\mathcal{B}} f(x)$ for $\mu$-almost every $x \in B_{0}$. Moreover, denote

$$
E_{\lambda}=E_{\lambda}^{f}=\left\{x \in \widehat{B}_{0}: M_{\mathcal{B}} f(x)>\lambda\right\}
$$

Lemma 4.1 If $B \in \mathcal{B}$ such that $m_{f}^{t}(B)>m_{f}^{t / \alpha}\left(\widehat{B}_{0}\right)$, then it holds that $r_{B} \leq \frac{\eta}{5} r_{B_{0}}$.

Proof By the assumption, we have

$$
t \mu(B) \leq \mu\left\{x \in B: f \geq m_{f}^{t}(B)\right\} \leq \mu\left\{x \in \widehat{B}_{0}: f>m_{f}^{t / \alpha}\left(\widehat{B}_{0}\right)\right\} \leq \frac{t}{\alpha} \mu\left(\widehat{B}_{0}\right) .
$$

Therefore, it holds that

$$
\alpha \leq \frac{\mu\left(\widehat{B}_{0}\right)}{\mu(B)} \leq c_{\mu}^{2}\left(\frac{r_{B_{0}}}{r_{B}}\right)^{D}=c_{\mu}^{2}(1+\eta)^{D}\left(\frac{r_{B_{0}}}{r_{B}}\right)^{D},
$$

which implies $r_{B} \leq \frac{\eta}{5} r_{B_{0}}$ by recalling (4.1).

The following lemma is a Calderón-Zygmund decomposition for medians in metric measure spaces with a doubling measure.

Lemma 4.2 Let $f \geq 0$ be a measurable function defined on $\widehat{B}_{0}$. Assume that $E_{\lambda} \neq \emptyset$ and

$$
m_{f}^{t / \alpha}\left(\widehat{B}_{0}\right) \leq \lambda
$$

holds for some $0<t \leq 1$, where $\alpha$ is given in (4.1). Then there exist countably many pairwise disjoint balls $B_{i} \in \mathcal{B}$ such that

(i) $\bigcup_{i} B_{i} \subset E_{\lambda} \subset \bigcup_{i} 5 B_{i}$,

(ii) $r_{B_{i}} \leq \frac{\eta}{5} r_{B_{0}}$,

(iii) $m_{f}^{t}\left(B_{i}\right)>\lambda$,

(iv) $m_{f}^{t}\left(\sigma B_{i}\right) \leq \lambda$ whenever $\sigma \geq 2$ and $\sigma B_{i} \in \mathcal{B}$.

The collection of balls $\left\{B_{i}\right\}_{i}$ is called the Calderón-Zygmund balls $B_{i, \lambda}$ at level $\lambda$. Furthermore, if $m_{f}^{t / \alpha}\left(\widehat{B}_{0}\right) \leq \lambda^{\prime} \leq \lambda$, then it is possible to choose Calderón-Zygmund balls $B_{j, \lambda^{\prime}}$ at level $\lambda^{\prime}$ in a manner that for each $B_{i, \lambda}$ we can find $B_{j, \lambda^{\prime}}$ such that $B_{i, \lambda} \subset 5 B_{j, \lambda^{\prime}}$.

Proof For every $x \in E_{\lambda}$, denote

$$
r_{x}(\lambda)=\sup \left\{r_{B}: B \in \mathcal{B}, x \in B, m_{f}^{t}(B)>\lambda\right\}
$$

By the assumption, the set over which the supremum is taken is non-empty. Moreover, Lemma 4.1 implies that $r_{x}(\lambda) \leq \frac{\eta}{5} r_{B_{0}}$. For every $x \in E_{\lambda}$, we can find a ball $B_{x, \lambda} \in \mathcal{B}$ with $x \in B_{x, \lambda}$ such that

$$
\frac{r_{x}(\lambda)}{2}<r_{B_{x, \lambda}} \leq r_{x}(\lambda) \text { and } m_{f}^{t}\left(B_{x, \lambda}\right)>\lambda
$$


We then have $m_{f}^{t}\left(\sigma B_{x, \lambda}\right) \leq \lambda$ whenever $\sigma \geq 2$ and $\sigma B_{x, \lambda} \in \mathcal{B}$. By applying the 5 -covering theorem, we obtain a countable collection of pairwise disjoint balls $\left\{B_{i}\right\}_{i}$ such that

$$
\bigcup_{i} B_{i} \subset E_{\lambda} \subset \bigcup_{i} 5 B_{i}
$$

Hence, the obtained balls $B_{i}$ are the Calderón-Zygmund balls at level $\lambda$ and we denote them by $B_{i, \lambda}$.

We have constructed the Calderón-Zygmund decomposition at level $\lambda$ and now focus on $\lambda^{\prime}$. Note that $E_{\lambda} \subset E_{\lambda^{\prime}}$ and $r_{x}(\lambda) \leq r_{x}\left(\lambda^{\prime}\right)$ for every $x \in E_{\lambda}$. Thus, for every $x \in E_{\lambda}$, we can choose a ball $B_{x, \lambda^{\prime}} \in \mathcal{B}$ with $x \in B_{x, \lambda^{\prime}}$ such that $B_{x, \lambda} \subset B_{x, \lambda^{\prime}}$,

$$
\frac{r_{x}\left(\lambda^{\prime}\right)}{2}<r_{B_{x, \lambda^{\prime}}} \leq r_{x}\left(\lambda^{\prime}\right) \text { and } m_{f}^{t}\left(B_{x, \lambda^{\prime}}\right)>\lambda^{\prime}
$$

Whereas, for every $x \in E_{\lambda^{\prime}} \backslash E_{\lambda}$, we choose the ball $B_{x, \lambda^{\prime}}$ in the similar way expect we do not have $B_{x, \lambda} \subset B_{x, \lambda^{\prime}}$.

We then apply the 5-covering theorem to the balls $B_{x, \lambda^{\prime}}$ to obtain the CalderónZygmund balls $B_{j, \lambda^{\prime}}$ at level $\lambda^{\prime}$. Moreover, the 5-covering theorem states that for every ball $B_{x, \lambda^{\prime}}$ there is an enlarged ball $5 B_{j, \lambda^{\prime}}$ such that $B_{x, \lambda^{\prime}} \subset 5 B_{j, \lambda^{\prime}}$. Since $B_{x, \lambda} \subset B_{x, \lambda^{\prime}}$ for every $x \in E_{\lambda}$, it holds that for each $B_{i, \lambda}$ there is $B_{j, \lambda^{\prime}}$ such that $B_{i, \lambda} \subset 5 B_{j, \lambda^{\prime}}$.

We move on to a good- $\lambda$ inequality which is crucial for the proof of the JohnNirenberg inequality. In the proof of the good- $\lambda$ type inequality for the integral-type $J N_{p}$ in [1], all Calderón-Zygmund balls can be treated in the same way. However, in the case of medians, we need to divide Calderón-Zygmund balls into two collections which must be considered separately. This is due to the fact that medians lack the monotonicity on sets that integrals have.

Lemma 4.3 Let $0<t \leq 1 / 2, K>1$ and $f \in J N_{p, 0, s}\left(\widehat{B}_{0}\right)$ for some $0<s \leq \frac{t}{2 K^{p} c_{\mu}^{3}}$. Assume that $E_{K \lambda} \neq \emptyset$ and

$$
m_{|f|}^{t / \alpha}\left(\widehat{B}_{0}\right) \leq \lambda
$$

where $\alpha$ is given in (4.1). Consider collections of Calderón-Zygmund balls $\left\{B_{i, \lambda}\right\}_{i}$ and $\left\{B_{j, K \lambda}\right\}_{j}$ for the function $|f|$ such that each $B_{j, K \lambda}$ is contained in some $5 B_{i, \lambda}$. Then it follows that

$$
\sum_{j=1}^{\infty} \mu\left(B_{j, K \lambda}\right) \leq \frac{2^{p} c_{\mu}^{3}}{(K-1)^{p}} \frac{\|f\|_{J N_{p, 0, s}\left(\widehat{B}_{0}\right)}^{p}}{\lambda^{p}}+\frac{1}{2 K^{p}} \sum_{i=1}^{\infty} \mu\left(B_{i, \lambda}\right) .
$$

Proof Denote

$$
J_{i}=\left\{j \in \mathbb{N}: B_{j, K \lambda} \subset 5 B_{i, \lambda}, j \notin \bigcup_{k=1}^{i-1} J_{k}\right\}
$$


for every $i \in \mathbb{N}$, and

$$
I=\left\{i \in \mathbb{N}: \mu\left(B_{i, \lambda}\right) \leq 2 K^{p} \mu\left(\bigcup_{j \in J_{i}} B_{j, K \lambda}\right)\right\}
$$

Particularly, the set $J_{i}$ contains those indexes $j \notin \bigcup_{k=1}^{i-1} J_{k}$ for which $B_{j, K \lambda}$ is contained in $5 B_{i, \lambda}$. Since every $B_{j, K \lambda}$ is contained in some $5 B_{i, \lambda}$, we get the partition

$$
\bigcup_{j=1}^{\infty} B_{j, K \lambda}=\bigcup_{i=1}^{\infty} \bigcup_{j \in J_{i}} B_{j, K \lambda}
$$

Using properties (ii), (v), (vii) of Lemma 2.6 and (iii), (iv) of Lemma 4.2 in this order, we obtain

$$
\begin{aligned}
m_{\left|f-m_{f}^{t}\left(5 B_{i, \lambda}\right)\right|}^{t}\left(B_{j, K \lambda}\right) & \geq m_{|f|-\left|m_{f}^{t}\left(5 B_{i, \lambda}\right)\right|}^{t}\left(B_{j, K \lambda}\right) \\
& =m_{|f|}^{t}\left(B_{j, K \lambda}\right)-\left|m_{f}^{t}\left(5 B_{i, \lambda}\right)\right| \\
& \geq m_{|f|}^{t}\left(B_{j, K \lambda}\right)-m_{|f|}^{t}\left(5 B_{i, \lambda}\right) \\
& \geq K \lambda-\lambda=(K-1) \lambda .
\end{aligned}
$$

Since $B_{j, K \lambda}$ are pairwise disjoint, property (x) of Lemma 2.6 implies that

$$
m_{\left|f-m_{f}^{t}\left(5 B_{i, \lambda}\right)\right|}^{t}\left(\bigcup_{j \in J_{i}} B_{j, K \lambda}\right) \geq(K-1) \lambda
$$

for every $i \in \mathbb{N}$. For $i \in I$, it holds that

$$
\mu\left(5 B_{i, \lambda}\right) \leq c_{\mu}^{3} \mu\left(B_{i, \lambda}\right) \leq 2 K^{p} c_{\mu}^{3} \mu\left(\bigcup_{j \in J_{i}} B_{j, K \lambda}\right) .
$$

Hence, by property (iii) of Lemma 2.6, we have

$$
(K-1) \lambda \leq m_{\left|f-m_{f}^{t}\left(5 B_{i, \lambda}\right)\right|}^{t}\left(\bigcup_{j \in J_{i}} B_{j, K \lambda}\right) \leq m_{\left|f-m_{f}^{t}\left(5 B_{i, \lambda}\right)\right|}^{t / \beta}\left(5 B_{i, \lambda}\right)
$$

for every $i \in I$, where $\beta=2 K^{p} c_{\mu}^{3}$.

Denote

$$
M_{\mathcal{B}}^{\#} f(x)=\sup _{\substack{B \ni x \\ B \in \mathcal{B}}} m_{\left|f-m_{f}^{t}(B)\right|}^{t / \beta}(B)
$$


Then for $i \in I$, we have $M_{\mathcal{B}}^{\#} f(x) \geq m_{\left|f-m_{f}^{t}\left(5 B_{i, \lambda}\right)\right|}^{t / \beta}\left(5 B_{i, \lambda}\right)>(K-1) \lambda$ for every $x \in 5 B_{i, \lambda}$. Thus, we get

$$
\mu\left(\bigcup_{i \in I} \bigcup_{j \in J_{i}} B_{j, K \lambda}\right) \leq \mu\left(\bigcup_{i \in I} 5 B_{i, \lambda}\right) \leq \mu\left(\left\{x \in \widehat{B}_{0}: M_{\mathcal{B}}^{\#} f(x)>(K-1) \lambda\right\}\right) .
$$

For every $x \in\left\{x \in \widehat{B}_{0}: M_{\mathcal{B}}^{\#} f(x)>(K-1) \lambda\right\}$, there exists $B_{x} \in \mathcal{B}$ such that $x \in B_{x}$ and $m_{\left|f-m_{f}^{t}\left(B_{x}\right)\right|}^{t / \beta}\left(B_{x}\right)>(K-1) \lambda$. Applying the 5-covering theorem, we get a countable collection of pairwise disjoint balls $B_{k}$ such that

$$
\left\{x \in \widehat{B}_{0}: M_{\mathcal{B}}^{\#} f(x)>(K-1) \lambda\right\} \subset \bigcup_{k=1}^{\infty} 5 B_{k} .
$$

We then have

$$
\begin{aligned}
\mu\left(\left\{x \in \widehat{B}_{0}: M_{\mathcal{B}}^{\#} f(x)>\right.\right. & (K-1) \lambda\}) \leq \sum_{k=1}^{\infty} \mu\left(5 B_{k}\right) \leq c_{\mu}^{3} \sum_{k=1}^{\infty} \mu\left(B_{k}\right) \\
& \leq c_{\mu}^{3} \frac{1}{(K-1)^{p} \lambda^{p}} \sum_{k=1}^{\infty} \mu\left(B_{k}\right)\left(m_{\left|f-m_{f}^{t}\left(B_{k}\right)\right|}^{t / \beta}\left(B_{k}\right)\right)^{p} \\
& \leq \frac{2^{p} c_{\mu}^{3}}{(K-1)^{p}} \frac{\|f\|_{J N_{p, 0, s}\left(\widehat{B}_{0}\right)}^{p}}{\lambda^{p}}
\end{aligned}
$$

whenever $s \leq \frac{t}{\beta}=\frac{t}{2 K^{p} c_{\mu}^{3}}$.

On the other hand, for $i \notin I$ we have

$$
\sum_{j \in J_{i}} \mu\left(B_{j, K \lambda}\right) \leq \frac{1}{2 K^{p}} \mu\left(B_{i, \lambda}\right),
$$

and thus summing over $i \notin I$ we get

$$
\sum_{i \notin I} \sum_{j \in J_{i}} \mu\left(B_{j, K \lambda}\right) \leq \frac{1}{2 K^{p}} \sum_{i \notin I} \mu\left(B_{i, \lambda}\right) .
$$

By combining the cases $i \in I$ and $i \notin I$, we conclude that

$$
\begin{aligned}
\sum_{j=1}^{\infty} \mu\left(B_{j, K \lambda}\right) & =\sum_{i \in I} \sum_{j \in J_{i}} \mu\left(B_{j, K \lambda}\right)+\sum_{i \notin I} \sum_{j \in J_{i}} \mu\left(B_{j, K \lambda}\right) \\
& \leq \frac{2^{p} c_{\mu}^{3}}{(K-1)^{p}} \frac{\|f\|_{J N_{p, 0, s}\left(\widehat{B}_{0}\right)}^{p}}{\lambda^{p}}+\frac{1}{2 K^{p}} \sum_{i=1}^{\infty} \mu\left(B_{i, \lambda}\right) .
\end{aligned}
$$


We now state our main result which is the John-Nirenberg inequality for $J N_{p, 0, s}$. It implies that $J N_{p, 0, s}(\widehat{B})$ is contained in $L^{p, \infty}(B)$ for all balls $B \subset X$.

Theorem 4.4 Let $0<s \leq s_{0}=\min \left\{\frac{1}{2 \alpha}, \frac{1}{8 c_{\mu}^{3}}\right\}$ and $s \leq r \leq \frac{1}{2}$, where $\alpha$ is given in (4.1). If $f \in J N_{p, 0, s}\left(\widehat{B}_{0}\right)$, then for every $\lambda>0$ it holds that

$$
\mu\left(\left\{x \in B_{0}:\left|f(x)-m_{f}^{r}\left(B_{0}\right)\right|>\lambda\right\}\right) \leq c \frac{\|f\|_{J N_{p, 0, s}\left(\widehat{B}_{0}\right)}^{p}}{\lambda^{p}},
$$

where the constant $c$ depends on $p$ and the doubling constant $c_{\mu}$, that is,

$$
c=\frac{2^{p+3} c_{\mu}^{6}}{\left(2^{\frac{1}{p}}-1\right)^{p}} .
$$

Proof Let $t=\frac{1}{2}$. We can assume that $E_{\lambda}=E_{\lambda}^{\left|f-m_{f}^{r}\left(B_{0}\right)\right|} \neq \emptyset$, since otherwise the claim is clear. In addition, we can assume that $\lambda>\lambda_{0}=m_{\left|f-m_{f}^{r}\left(B_{0}\right)\right|}^{t / \alpha}\left(\widehat{B}_{0}\right)$, since otherwise if $0<\lambda \leq \lambda_{0}$, then by the trivial estimate and Lemma 3.2 we have

$$
\begin{aligned}
\mu\left(\left\{x \in B_{0}:\left|f(x)-m_{f}^{r}\left(B_{0}\right)\right|>\lambda\right\}\right) & \leq \mu\left(\widehat{B}_{0}\right) \frac{\left(m_{\left|f-m_{f}^{r}\left(B_{0}\right)\right|}^{t / \alpha}\left(\widehat{B}_{0}\right)\right)^{p}}{\lambda^{p}} \\
& \leq 2^{p} \frac{\|f\|_{J N_{p, 0, s}}^{p}}{\lambda^{p}}
\end{aligned}
$$

whenever $s \leq \frac{t}{\alpha}=\frac{1}{2 \alpha}$ and $s \leq r \leq \frac{1}{2}$. Thus, the conditions in Lemma 4.2 and Lemma 4.3 hold for the function $\left|f-m_{f}^{r}\left(B_{0}\right)\right|$.

Let $K=2^{1 / p}$ and $N \in \mathbb{N}$ such that

$$
K^{N} \lambda_{0}<\lambda \leq K^{N+1} \lambda_{0}
$$

Consider $N+1$ families of Calderón-Zygmund balls at levels $\lambda_{0}, K \lambda_{0}, \ldots, K^{N} \lambda_{0}$. Note that for every $n=0,1, \ldots, N-1$, each $B_{i, K^{n+1} \lambda}$ is contained in some $5 B_{j, K^{n} \lambda}$. It follows that

$$
\begin{aligned}
\mu\left(\left\{x \in B_{0}:\left|f(x)-m_{f}^{r}\left(B_{0}\right)\right|>\lambda\right\}\right) & \leq \mu\left(\left\{x \in B_{0}:\left|f(x)-m_{f}^{r}\left(B_{0}\right)\right|>K^{N} \lambda_{0}\right\}\right) \\
& \leq \sum_{j=1}^{\infty} \mu\left(5 B_{j, K^{N} \lambda_{0}}\right) \leq c_{\mu}^{3} \sum_{j=1}^{\infty} \mu\left(B_{j, K^{N} \lambda_{0}}\right) .
\end{aligned}
$$

We claim that

$$
\sum_{i=1}^{\infty} \mu\left(B_{i, K^{n} \lambda_{0}}\right) \leq c_{1} \frac{\|f\|_{J N_{p, 0, s}}^{p}}{\left(K^{n} \lambda_{0}\right)^{p}}
$$


for every $n=0,1, \ldots, N$, where

$$
c_{1}=\frac{2^{p+1} c_{\mu}^{3} K^{p}}{(K-1)^{p}} .
$$

We prove the claim by induction. First, note that the claim holds for $n=0$ since

$$
\sum_{i=1}^{\infty} \mu\left(B_{i, \lambda_{0}}\right) \leq \mu\left(\widehat{B}_{0}\right)=\mu\left(\widehat{B}_{0}\right) \frac{\left(m_{\left|f-m_{f}^{r}\left(B_{0}\right)\right|}^{t / \alpha}\left(\widehat{B}_{0}\right)\right)^{p}}{\lambda_{0}^{p}} \leq 2^{p} \frac{\|f\|_{J N_{p, 0, s}}^{p}}{\lambda_{0}^{p}} .
$$

Assume then that the claim holds for $k \in\{0,1, \ldots, N-1\}$, that is,

$$
\sum_{i=1}^{\infty} \mu\left(B_{i, K^{k} \lambda_{0}}\right) \leq c_{1} \frac{\|f\|_{J N_{p, 0, s}}^{p}}{\left(K^{k} \lambda_{0}\right)^{p}} .
$$

We show that this implies the claim for $k+1$. By using Lemma 4.3 for $K^{k} \lambda_{0}$, we observe that

$$
\begin{aligned}
\sum_{j=1}^{\infty} \mu\left(B_{j, K^{k+1} \lambda_{0}}\right) & \leq \frac{2^{p} c_{\mu}^{3}}{(K-1)^{p}} \frac{\|f\|_{J N_{p, 0, s}}^{p}}{\left(K^{k} \lambda_{0}\right)^{p}}+\frac{1}{2 K^{p}} \sum_{i=1}^{\infty} \mu\left(B_{i, K^{k} \lambda_{0}}\right) \\
& \leq \frac{2^{p} c_{\mu}^{3}}{(K-1)^{p}} \frac{\|f\|_{J N_{p, 0, s}}^{p}}{\left(K^{k} \lambda_{0}\right)^{p}}+\frac{c_{1}}{2 K^{p}} \frac{\|f\|_{J N_{p, 0, s}}^{p}}{\left(K^{k} \lambda_{0}\right)^{p}} \\
& =\left(\frac{2^{p} c_{\mu}^{3} K^{p}}{(K-1)^{p}}+\frac{c_{1}}{2}\right) \frac{\|f\|_{J N_{p, 0, s}}^{p}}{\left(K^{k+1} \lambda_{0}\right)^{p}} \\
& =c_{1} \frac{\|f\|_{J N_{p, 0, s}}^{p}}{\left(K^{k+1} \lambda_{0}\right)^{p}}
\end{aligned}
$$

Therefore, the claim holds for $k+1$.

Hence, we conclude that

$$
\begin{aligned}
\mu\left(\left\{x \in B_{0}:\left|f(x)-m_{f}^{r}\left(B_{0}\right)\right|>\lambda\right\}\right) & \leq c_{\mu}^{3} c_{1} \frac{\|f\|_{J N_{p, 0, s}}^{p}}{\left(K^{N} \lambda_{0}\right)^{p}} \\
& =c_{\mu}^{3} c_{1} K^{p} \frac{\|f\|_{J N_{p, 0, s}}^{p}}{\left(K^{N+1} \lambda_{0}\right)^{p}} \\
& \leq c \frac{\|f\|_{J N_{p, 0, s}}^{p}}{\lambda^{p}}
\end{aligned}
$$

where

$$
c=c_{\mu}^{3} c_{1} K^{p}=\frac{2^{p+1} c_{\mu}^{6} K^{2 p}}{(K-1)^{p}}=\frac{2^{p+3} c_{\mu}^{6}}{\left(2^{\frac{1}{p}}-1\right)^{p}},
$$


since $K=2^{1 / p}$.

\section{Global John-Nirenberg Inequality for $J N_{p, 0, s}$ in Boman Sets}

We give a proof for the global John-Nirenberg inequality for $J N_{p, 0, s}$ in Boman sets. For more detailed discussion about Boman sets, see [32] and references therein.

Definition 5.1 A set $\Delta \subset X$ is called Boman if there are constants $C_{2}>C_{1}>1$, $C_{3}>1, \rho>1$ and $M \in \mathbb{N}$ and a collection of pairwise disjoint balls $\mathcal{F}$ such that

(i) $\Delta=\bigcup_{B \in \mathcal{F}} C_{1} B=\bigcup_{B \in \mathcal{F}} C_{2} B$.

(ii) If $B \in \mathcal{F}$, there are at most $M$ balls $V \in \mathcal{F}$ with $C_{2} V \cap C_{2} B \neq \emptyset$.

(iii) There is a central ball $B_{*} \in \mathcal{F}$ such that for each $B \in \mathcal{F}$ there exists a finite collection of balls $\mathcal{C}(B)=\left\{B_{i}\right\}_{i=1}^{k_{B}} \subset \mathcal{F}$ with $B_{1}=B_{*}$ and $B_{k_{B}}=B$.

(iv) In $\mathcal{C}(B)$, for each pair of balls $B_{i}$ and $B_{i-1}$ corresponding to consecutive indices there exists a ball $D_{i} \subset C_{1} B_{i} \cap C_{1} B_{i-1}$ such that $\mu\left(D_{i}\right) \geq C_{3}\left(\mu\left(B_{i}\right)+\mu\left(B_{i-1}\right)\right)$.

(v) If $V \in \mathcal{C}(B)$, then $B \subset \rho V$.

Parameters $C_{1}, C_{2}, C_{3}, \rho$ and $M$ in the results below are the same as in Definition 5.1. The proof of the following lemma can be found in [32] for integral averages. The proof is identical for medians, and thus is omitted here.

Lemma 5.2 Let $\Delta \subset X$ be a Boman set, $1<p<\infty$ and $0<s \leq 1 / 2$. Then

$$
\sum_{B \in \mathcal{F}}\left\|m_{f}^{s}\left(C_{1} B\right)-m_{f}^{s}\left(C_{1} B_{*}\right)\right\|_{L^{p, \infty}\left(C_{1} B\right)}^{p} \leq C_{0} \sum_{V \in \mathcal{F}}\left\|f-m_{f}^{s}\left(C_{1} V\right)\right\|_{L^{p, \infty}\left(C_{1} V\right)}^{p},
$$

where the constant $C_{0}$ depends on $p$, the doubling constant $c_{\mu}, C_{1}, C_{2}, C_{3}, \rho$ and $M$.

For the next theorem, the global John-Nirenberg lemma, we fix the parameter $\eta$ from Sect. 4 such that $1+\eta=\frac{C_{2}}{C_{1}}$. The proof follows that of [32].

Theorem 5.3 Let $\Delta \subset X$ be a Boman set and $0<s \leq s_{0}$, where $s_{0}$ is given in Theorem 4.4. If $f \in J N_{p, 0, s}(\Delta)$, then there exists $a \in \mathbb{R}$ such that for every $\lambda>0$ it holds that

$$
\mu(\{x \in \Delta:|f(x)-a|>\lambda\}) \leq C \frac{\|f\|_{J N_{p, 0, s}(\Delta)}^{p}}{\lambda^{p}},
$$

where $C$ depends on $p$, the doubling constant $c_{\mu}, C_{1}, C_{2}, C_{3}, \rho$ and $M$. 
Proof Let $s \leq r \leq \frac{1}{2}, \mathcal{F}$ be the collection of balls in the definition of the Boman set $\Delta$ and $B_{*}$ be the central ball. We have

$$
\begin{aligned}
\mu\left(\left\{x \in \Delta:\left|f-m_{f}^{r}\left(C_{1} B_{*}\right)\right|>\lambda\right\}\right) \leq \sum_{B \in \mathcal{F}} \mu\left(\left\{x \in C_{1} B:\left|f-m_{f}^{r}\left(C_{1} B_{*}\right)\right|>\lambda\right\}\right) \\
\leq \sum_{B \in \mathcal{F}} \mu\left(\left\{x \in C_{1} B:\left|f-m_{f}^{r}\left(C_{1} B\right)\right|>\lambda / 2\right\}\right) \\
\quad+\sum_{B \in \mathcal{F}} \mu\left(\left\{x \in C_{1} B:\left|m_{f}^{r}\left(C_{1} B\right)-m_{f}^{r}\left(C_{1} B_{*}\right)\right|>\lambda / 2\right\}\right) \\
=I_{1}+I_{2} .
\end{aligned}
$$

By applying Theorem 4.4, we get

$$
I_{1} \leq 2^{p} c \sum_{B \in \mathcal{F}} \frac{\|f\|_{J N_{p, 0, s}\left(C_{2} B\right)}^{p} .}{\lambda^{p}} .
$$

To estimate the second term, we use the definition of weak $L^{p}$ norm, Lemma 5.2 and Theorem 4.4 to obtain

$$
\begin{aligned}
\left(\frac{\lambda}{2}\right)^{p} I_{2} & \leq \sum_{B \in \mathcal{F}}\left\|m_{f}^{r}\left(C_{1} B\right)-m_{f}^{r}\left(C_{1} B_{*}\right)\right\|_{L^{p, \infty}\left(C_{1} B\right)}^{p} \\
& \leq C_{0} \sum_{B \in \mathcal{F}}\left\|f-m_{f}^{r}\left(C_{1} B\right)\right\|_{L^{p, \infty}\left(C_{1} B\right)}^{p} \\
& =C_{0} \sum_{B \in \mathcal{F}} \sup _{\gamma>0} \gamma^{p} \mu\left(\left\{x \in C_{1} B:\left|f-m_{f}^{r}\left(C_{1} B\right)\right|>\gamma\right\}\right) \\
& \leq C_{0} \sum_{B \in \mathcal{F}} c\|f\|_{J N_{p, 0, s}\left(C_{2} B\right)}^{p} .
\end{aligned}
$$

Therefore, it holds that

$$
I_{1}+I_{2} \leq \frac{\widetilde{C}}{\lambda^{p}} \sum_{B \in \mathcal{F}}\|f\|_{J N_{p, 0, s}\left(C_{2} B\right)}^{p},
$$

where $\widetilde{C}=2^{p} c\left(C_{0}+1\right)$. By property (ii) of Lemma 5.1, the collection $\left\{C_{2} B\right\}_{B \in \mathcal{F}}$ consists of balls that intersect at most $M$ balls of the same collection. Thus, it can be decomposed into at most $M$ collections of pairwise disjoint balls $\mathcal{D}_{i}, i=1, \ldots, M$ 
such that $\left\{C_{2} B\right\}_{B \in \mathcal{F}}=\bigcup_{i=1}^{M} \mathcal{D}_{i}$. This implies that

$$
\begin{aligned}
\mu\left(\left\{x \in \Delta:\left|f-m_{f}^{r}\left(C_{1} B_{*}\right)\right|>\lambda\right\}\right) & \leq \frac{\widetilde{C}}{\lambda^{p}} \sum_{B \in \mathcal{F}}\|f\|_{J N_{p, 0, s}\left(C_{2} B\right)}^{p} \\
& =\frac{\widetilde{C}}{\lambda^{p}} \sum_{i=1}^{M} \sum_{B \in \mathcal{D}_{i}}\|f\|_{J N_{p, 0, s}\left(C_{2} B\right)}^{p} \\
& \leq \frac{C}{\lambda^{p}}\|f\|_{J N_{p, 0, s}(\Delta)}^{p},
\end{aligned}
$$

where $C=\widetilde{C} M$. This concludes the proof.

If all balls are Boman sets with uniform parameters, then the median-type JohnNirenberg space coincides with the integral-type John-Nirenberg space in every open set. For example, geodesic spaces satisfy the uniform Boman condition on balls [16].

Corollary 5.4 Let $1<p<\infty, 0<q<p$ and $0<s \leq s_{0}$, where $s_{0}$ is given in Theorem 4.4. Assume that all balls in $X$ are Boman sets with uniform parameters $C_{1}, C_{2}, C_{3}, \rho$ and $M$. Then for every open set $\Omega \subset X$ it holds that

$$
s^{\frac{1}{q}}\|f\|_{J N_{p, 0, s}(\Omega)} \leq\|f\|_{J N_{p, q}(\Omega)} \leq\left(\frac{C p}{p-q}\right)^{\frac{1}{q}}\|f\|_{J N_{p, 0, s}(\Omega)},
$$

where $C$ is the constant from Theorem 5.3.

Proof Let $\left\{B_{i}\right\}_{i}$ be a countable collection of pairwise disjoint balls contained in $\Omega$. The first inequality is stated and proven in Proposition 3.4. For the second inequality, since by the assumption the balls $B_{i}$ are Boman sets, we may apply Theorem 5.3 on $B_{i}$. This together with Cavalieri's principle implies

$$
\begin{aligned}
\int_{B_{i}}|f-a|^{q} d \mu= & q \int_{0}^{\infty} \lambda^{q-1} \mu\left(\left\{x \in B_{i}:|f-a|>\lambda\right\}\right) d \lambda \\
\leq & q \int_{\mu\left(B_{i}\right)^{-\frac{1}{p}}\|f\|_{J N_{p, 0, s}\left(B_{i}\right)}^{\infty} C \lambda^{q-p-1}\|f\|_{J N_{p, 0, s}\left(B_{i}\right)}^{p} d \lambda} \\
& +q \int_{0}^{\mu\left(B_{i}\right)^{-\frac{1}{p}}\|f\|_{J N_{p, 0, s}\left(B_{i}\right)}} \lambda^{q-1} \mu\left(B_{i}\right) d \lambda \\
= & \frac{C q}{p-q} \mu\left(B_{i}\right)^{1-\frac{q}{p}}\|f\|_{J N_{p, 0, s}\left(B_{i}\right)}^{q}+\mu\left(B_{i}\right)^{1-\frac{q}{p}}\|f\|_{J N_{p, 0, s}\left(B_{i}\right)}^{q} \\
\leq & \frac{C p}{p-q} \mu\left(B_{i}\right)^{1-\frac{q}{p}}\|f\|_{J N_{p, 0, s}\left(B_{i}\right)}^{q},
\end{aligned}
$$


where $C$ is the constant from Theorem 5.3. We then estimate

$$
\begin{aligned}
\sum_{i=1}^{\infty} \mu\left(B_{i}\right)\left(\inf _{c_{i}} f_{B_{i}}\left|f-c_{i}\right|^{q} d \mu\right)^{\frac{p}{q}} & \leq \sum_{i=1}^{\infty} \mu\left(B_{i}\right)\left(f_{B_{i}}|f-a|^{q} d \mu\right)^{\frac{p}{q}} \\
& \leq\left(\frac{C p}{p-q}\right)^{\frac{p}{q}} \sum_{i=1}^{\infty}\|f\|_{J N_{p, 0, s}\left(B_{i}\right)}^{p} \\
& \leq\left(\frac{C p}{p-q}\right)^{\frac{p}{q}}\|f\|_{J N_{p, 0, s}(\Omega)}^{p} .
\end{aligned}
$$

Thus, we conclude that

$$
\|f\|_{J N_{p, q}(\Omega)} \leq\left(\frac{C p}{p-q}\right)^{\frac{1}{q}}\|f\|_{J N_{p, 0, s}(\Omega)} .
$$

Acknowledgements The author would like to thank Juha Kinnunen and Riikka Korte for valuable discussions. The author would also like to thank the anonymous referee for carefully reading the paper and for constructive comments. The research was supported by the Academy of Finland.

Funding Open Access funding provided by Aalto University.

Open Access This article is licensed under a Creative Commons Attribution 4.0 International License, which permits use, sharing, adaptation, distribution and reproduction in any medium or format, as long as you give appropriate credit to the original author(s) and the source, provide a link to the Creative Commons licence, and indicate if changes were made. The images or other third party material in this article are included in the article's Creative Commons licence, unless indicated otherwise in a credit line to the material. If material is not included in the article's Creative Commons licence and your intended use is not permitted by statutory regulation or exceeds the permitted use, you will need to obtain permission directly from the copyright holder. To view a copy of this licence, visit http://creativecommons.org/licenses/by/4.0/.

\section{References}

1. Aalto, D., Berkovits, L., Kansanen, O.E., Yue, H.: John-Nirenberg lemmas for a doubling measure. Stud. Math. 204(1), 21-37 (2011)

2. Berkovits, L., Kinnunen, J., Martell, J.M.: Oscillation estimates, self-improving results and good- $\lambda$ inequalities. J. Funct. Anal 270(9), 3559-3590 (2016)

3. Björn, A., Björn, J.: Nonlinear Potential Theory on Metric Spaces, EMS Tracts in Mathematics, vol. 17. European Mathematical Society (EMS), Zürich (2011)

4. Boman, J.: $\mathrm{L}_{p}$-estimates for very strongly elliptic systems, Report no. 29, Department of Mathematics, University of Stockholm, Sweden (1982)

5. Buckley, S., Koskela, P., Guozhen, L.: Boman Equals John, XVIth Rolf Nevanlinna Colloquium (Joensuu, 1995), pp. 91-99. de Gruyter, Berlin (1996)

6. Campanato, S.: Su un teorema di interpolazione di G. Stampacchia. Ann. Scuola Norm. Sup. Pisa Cl. Sci. (3) 20, 649-652 (1966)

7. Chua, S.-K.: Weighted Sobolev inequalities on domains satisfying the chain condition. Proc. Am. Math. Soc. 117(2), 449-457 (1993)

8. Dafni, G., Hytönen, T., Korte, R., Yue, H.: The space $J N_{p}$ : nontriviality and duality. J. Funct. Anal. 275(3), 577-603 (2018)

9. Federer, H., Ziemer, W.P.: The Lebesgue set of a function whose distribution derivatives are p-th power summable. Indiana Univ. Math. J. 22(73), 139-158 (1972) 
10. Franchi, B., Pérez, C., Wheeden, R.L.: Self-improving properties of John-Nirenberg and Poincaré inequalities on spaces of homogeneous type. J. Funct. Anal. 153(1), 108-146 (1998)

11. Fujii, N.: A condition for a two-weight norm inequality for singular integral operators. Stud. Math. 98(3), 175-190 (1991)

12. Giaquinta, M.: Introduction to Regularity Theory for Nonlinear Elliptic Systems. Lectures in Mathematics ETH Zürich. Birkhäuser Verlag, Basel (1993)

13. Giaquinta, M., Martinazzi, L.: An Introduction to the Regularity Theory for Elliptic Systems, Harmonic Maps and Minimal Graphs, second ed., Appunti. Scuola Normale Superiore di Pisa (Nuova Serie) [Lecture Notes. Scuola Normale Superiore di Pisa (New Series)], vol. 11, Edizioni della Normale, Pisa (2012)

14. Giusti, E.: Direct Methods in the Calculus of Variations. World Scientific Publishing Co. Inc., River Edge, NJ (2003)

15. Gogatishvili, A., Koskela, P., Zhou, Y.: Characterizations of Besov and Triebel-Lizorkin spaces on metric measure spaces. Forum Math. 25(4), 787-819 (2013)

16. Hajłasz, P., Koskela, P.: Sobolev met Poincaré, Mem. Am. Math. Soc. 145 (2000), no. 688, x+101

17. Heikkinen, T.: Generalized Lebesgue points for Hajłasz functions. J. Funct. Spaces (2018). Art. ID $5637042,12 \mathrm{pp}$

18. Heikkinen, T., Ihnatsyeva, L., Tuominen, H.: Measure density and extension of Besov and TriebelLizorkin functions. J. Fourier Anal. Appl. 22(2), 334-382 (2016)

19. Heikkinen, T., Kinnunen, J.: A median approach to differentiation bases. Atti Accad. Naz. Lincei Rend. Lincei Mat. Appl. 30(1), 41-66 (2019)

20. Heikkinen, T., Koskela, P., Tuominen, H.: Approximation and quasicontinuity of Besov and TriebelLizorkin functions. Trans. Am. Math. Soc. 369(5), 3547-3573 (2017)

21. Heikkinen, T., Tuominen, H.: Approximation by Hölder functions in Besov and Triebel-Lizorkin spaces. Constr. Approx. 44(3), 455-482 (2016)

22. Heinonen, J.: Lectures on Analysis on Metric Spaces. Universitext, Springer, New York (2001)

23. Hurri-Syrjänen, R., Marola, N., Vähäkangas, A.V.: Aspects of local-to-global results. Bull. Lond. Math. Soc. 46(5), 1032-1042 (2014)

24. Jawerth, B., Pérez, C., Welland, G.: The positive cone in Triebel-Lizorkin spaces and the relation among potential and maximal operators. In: Milman, M., Schonbek, T. (eds.) Harmonic Analysis and Partial Differential Equations (Boca Raton, FL, 1998). Contemporary Mathematics, vol. 107, pp. 71-91. American Mathematical Society, Providence, RI (1990)

25. Jawerth, B., Torchinsky, A.: Local sharp maximal functions. J. Approx. Theory 43(3), 231-270 (1985)

26. John, F.: Quasi-isometric mappings, Seminari 1962/63 Anal. Alg. Geom. e Topol., vol. 2, pp. 462-473, Ist. Naz. Alta Mat, Ediz. Cremonese, Rome (1965)

27. John, F., Nirenberg, L.: On functions of bounded mean oscillation. Commun. Pure Appl. Math. 14, 415-426 (1961)

28. Karak, N.: Triebel-Lizorkin capacity and Hausdorff measure in metric spaces. Math. Slovaca 70(3), 617-624 (2020)

29. Lerner, A.K.: A pointwise estimate for the local sharp maximal function with applications to singular integrals. Bull. Lond. Math. Soc. 42(5), 843-856 (2010)

30. Lerner, A.K., Pérez, C.: Self-improving properties of generalized Poincaré type inequalities through rearrangements. Math. Scand. 97(2), 217-234 (2005)

31. MacManus, P., Pérez, C.: Generalized Poincaré inequalities: sharp self-improving properties. Int. Math. Res. Not. 2, 101-116 (1998)

32. Marola, N., Saari, O.: Local to global results for spaces of BMO type. Math. Z. 282(1-2), 473-484 (2016)

33. Poelhuis, J., Torchinsky, A.: Medians, continuity, and vanishing oscillation. Stud. Math. 213(3), 227242 (2012)

34. Stampacchia, G.: The spaces $\mathcal{L}^{(p, \lambda)}, N^{(p, \lambda)}$ and interpolation. Ann. Scuola Norm. Sup. Pisa Cl. Sci. (3) 3, 443-462 (1965)

35. Strömberg, J.-O.: Bounded mean oscillation with Orlicz norms and duality of Hardy spaces. Indiana Univ. Math. J. 28(3), 511-544 (1979)

36. Strömberg, J.-O., Torchinsky, A.: Weighted Hardy Spaces. Lecture Notes in Mathematics, vol. 1381. Springer, Berlin (1989)

37. Zhou, Y.: Fractional Sobolev extension and imbedding. Trans. Am. Math. Soc. 367(2), 959-979 (2015) 
Publisher's Note Springer Nature remains neutral with regard to jurisdictional claims in published maps and institutional affiliations. 